\title{
Quantitative Electroencephalographic Analysis of Delirium Tremens Development Following Alcohol- withdrawal Seizure
}

Hee-Jung Mo

Hallym University Dongtan Sacred Heart Hospital

Jee-Eun Yoon

Uijeongbu Eulji Medical Center

Dong Wook Kim

Konkuk University Hospital, Konkuk University

Hee-Jin Im ( $\nabla$ coolere@naver.com )

Hallym University Dongtan Sacred Heart Hospital

\section{Research Article}

Keywords: Alcohol-withdrawal seizure, Delirium tremens, Quantitative EEG, Spectral analysis

Posted Date: December 20th, 2021

DOI: https://doi.org/10.21203/rs.3.rs-1146221/v1

License: (a) (i) This work is licensed under a Creative Commons Attribution 4.0 International License.

Read Full License 


\section{Abstract}

Seizures and delirium tremens (DTs) are recognized as severe alcohol-withdrawal symptoms. Prolonged admission and serious complications associated with alcohol-withdrawal are responsible for increased costs and use of medical and social resources. We compared differences in quantitative electroencephalography (EEG) in patients after alcohol-withdrawal seizures (AWS; $n=13$ ), performed in the intensive care unit within $48 \mathrm{~h}$ of admission, and in age- and sex-matched healthy controls. We also investigated the prognostic value of quantitative EEG, for the development of alcohol DTs after AWS in a retrospective, case-control study. The spectral power of each band frequency and the ratio of the theta to alpha band (TAR) in the electroencephalogram were analysed using iSyncBrain ${ }^{\circledR}$ (iMediSync, Inc., Korea). The beta frequency and the alpha frequency band power were significantly higher and lower, respectively, in patients than in age- and sex-matched healthy controls. In AWS patients with DTs, the relative beta3 power was lower, particularly in the left frontal area, and the TAR was significantly higher in the central channel than in those without DTs. Quantitative EEG showed neuronal excitability and decreased cognitive activities characteristic of AWS patients associated with alcohol withdrawal state and we demonstrated that quantitative EEG also might be a helpful tool for detecting patients at high risk of developing DTs during an alcohol-dependence period.

\section{Introduction}

Alcohol-withdrawal seizures (AWS) occur in alcohol-dependent individuals, typically $1-3$ days after the last drink, peak at about 48 hours, and are markedly reduced by day $5-7$ of abstinence ${ }^{1}$.

As a rebound phenomenon, AWS are linked to the abrupt cessation of prolonged intoxication and alcohol abuse. Alcohol acts on the brain via several mechanisms. During alcohol abstinence, NMDA receptor function is enhanced, GABAergic transmission is reduced, and the dopaminergic system is dysregulated, all of which lead to withdrawal symptoms and signs ${ }^{2}$. Alcohol-withdrawal symptoms include tremor, insomnia, nausea or vomiting, transient hallucinations or illusions, psychomotor agitation, anxiety, and seizures. Seizures and delirium are recognized as severe forms of alcohol-withdrawal symptoms. Previous reviews showed that $4-15 \%$ of people with alcohol dependency (and up to $20-30 \%$ in admitted patients) develop DTs or seizures ${ }^{3}$. Withdrawal seizures may occur $12-48 \mathrm{~h}$ after the last drink, and sequentially some develop delirium tremens (DTs) within 48-72 $h^{4}$. DTs, also called withdrawal delirium, is an abrupt onset of fluctuating confusion, disorientation, and encephalopathic conditions, including hallucinations. It is characterized by severe hyperadrenergic and dysautonomic states, including hyperthermia, cardiac arrhythmias, complications of withdrawal seizures, or concomitant medical disorders, which can lead to death in approximately $1-4 \%$ of hospitalized patients ${ }^{4,5}$. The mortality in the 8 years following an episode of DTs is $30.8 \%$ (hazard ratio $1.38,95 \%$ confidence interval $0.43-4.48$ ), which is comparable to that in patients with severe malignant diseases ${ }^{6}$.

Clinically significant predictors for the occurrence of DTs, such as a history of DTs history, lower serum potassium, a lower platelet count, the presence of structural brain lesions, and a high pulse rate above 
$100-120 \mathrm{bpm}^{7,8}$. In previous reports, admission with seizures per se and older age ( $>70$ years) increased the risk of hallucinations and delirium as a withdrawal complication in general hospitals. The biggest hazard for delirium is an increased number of days since the last drink ${ }^{9,10}$. However, previous studies have mainly focused on predicting the severity of alcohol-withdrawal symptoms, particularly seizures, and differed in their methodology. Thus, there is no general consensus on which factors increase the risk of DTs.

A predictive tool for the risk of DTs development after AWS would assist clinicians in making therapeutic decisions and thereby reduce patient mortality as well as the socioeconomic burden associated with alcoholism. Quantitative electroencephalography (QEEG) allows the analysis of quantitative features of brain function via oscillatory electrophysiological rhythms, such as spectral power and coherence. Recently, various reports have emerged regarding the role of potential biomarkers of cognitive function in dementia ${ }^{11}$. A previous study on delirium detection using QEEG showed significantly increased theta and delta, and decreased alpha activity in delirium patients ${ }^{12,13}$, and the related delta power in the frontalparietal electrode pair between patients with and without delirium who underwent cardiothoracic surgery ${ }^{13}$.

In this study, we aimed to evaluate the differences in QEEG between patients who have had AWS and healthy controls, and to investigate early changes of QEEG for the development of alcohol-related DTs after AWS.

\section{Results}

\section{Demographic and clinical characteristics of patients}

Consecutive patients who visited the emergency department between March 2018 and December 2020 for acute seizures, deemed to be related to alcohol-withdrawal by a physician, were retrospectively included in the study.

Thirteen patients were finally enrolled in the study. The mean age of participants was $50.1 \pm 10.7$ years and all patients were male. DTs occurred in eight of the 13 patients (61.5\%). Most of them (10 of 13 patients, $76.9 \%$ ) experienced their first-ever seizure at admission; for the others, all previous seizures occurred during the alcohol-withdrawal state. AWS occurred on average $31.5 \pm 21.5 \mathrm{~h}$ after the last selfreported drinking by participants. The average daily amount of alcohol consumption was $160.1 \pm 65.9$ g/day.

The demographics and clinical characteristics of the patients with and without DTs are shown in Table 1. There were no significant differences in demographic and clinical alcohol-related factors, such as age, sex, history of AWS, average amount of alcohol consumed, and time interval from the last drink to seizure between the two groups. Laboratory tests also did not show statistically significant differences between the groups. 
Table 1

Demographic and clinical characteristics of patients and control group.

\begin{tabular}{|c|c|c|c|}
\hline Characteristics & $\begin{array}{l}\text { DT (+) } \\
(\mathrm{N}=8)\end{array}$ & $\begin{array}{l}\text { DT }(-) \\
(\mathrm{N}=5)\end{array}$ & $p$-value \\
\hline Age (y) & $47.6 \pm 10.7$ & $54.0 \pm 10.5$ & 0.061 \\
\hline Male & $8(100.0)$ & $5(100.0)$ & 0.692 \\
\hline Previous alcohol withdrawal seizure (Yes) & $3(37.5)$ & $0(0)$ & 0.581 \\
\hline \multicolumn{4}{|l|}{ Last drink before seizure } \\
\hline Less than hour & $3(37.5)$ & $1(20.0)$ & \multirow[t]{3}{*}{0.762} \\
\hline $24-72$ hour & $4(50.0)$ & $3(60.0)$ & \\
\hline More than 72 hour & $1(12.5)$ & $1(20.0)$ & \\
\hline Alcohol intake (g/day) & $163.0 \pm 78.0$ & $155.5 \pm 48.2$ & 0.883 \\
\hline Platelets (g/l) & $139.8 \pm 86.0$ & $137.2 \pm 59.6$ & 0.943 \\
\hline Y-GT (mmol/l) & $436.0 \pm 364.5$ & $1056.6 \pm 481.7$ & 0.065 \\
\hline $\mathrm{Na}+(\mathrm{mmol} / \mathrm{l})$ & $138.0 \pm 3.7$ & $138.8 \pm 3.6$ & 0.943 \\
\hline $\mathrm{K}+(\mathrm{mmol} / \mathrm{l})$ & $3.9 \pm 0.4$ & $3.5 \pm 0.8$ & 0.185 \\
\hline BUN (mg/dl) & $10.0 \pm 0.4$ & $6.8 \pm 2.8$ & 0.107 \\
\hline Creatinine (mg/dl) & $0.9 \pm 0.2$ & $0.9 \pm 0.1$ & 0.622 \\
\hline \multicolumn{4}{|c|}{ The data are presented as the mean \pm the standard deviation or as the number (\%). } \\
\hline${ }^{*} \mathrm{p}<0.05$, based on the Mann-Whitney $U \mathrm{t} \epsilon$ & or Fisher test ${ }^{\beta}$ & & \\
\hline
\end{tabular}

\section{Comparison of spectral power pattern between alcohol- withdrawal patients and healthy controls}

We performed a relative and absolute sensor-level analysis between the AWS patients and the age- and sex-matched healthy adult group.

In the absolute spectral power analysis, the AWS patients showed lower alpha-2 power in the bilateral temporal and occipital areas, and higher beta- 1 and beta- 2 power in the central $(\mathrm{Cz})$ area than the healthy controls $(P<0.05)$. 
In the relative power analysis of the AWS patients, the relative alpha-1 power in the frontocentral area and the alpha-2 power in virtually the entire cortex, except for the parietal area, were lower in the the AWS group than in healthy control group (Figure $2 \mathrm{~A}-\mathrm{B}, \mathrm{P}<0.05$ ).

In contrast, the relative delta power was higher in the frontal and temporo-parieto-occipital areas (Figure $2 C, P<0.05$ ), and the all range of beta power (beta-1, beta-2, and beta-3) was higher in the frontal and central areas in the the AWS group than in the normal controls (Figure 2D-F, $P<0.05$ ).

\section{Comparison of spectral power pattern between the patients with and without DTs}

To identify the difference in the spectral power pattern and find early predictive features of DT, we compared the QEEG performed immediately (within $48 \mathrm{~h}$ ) after AWS between the groups with $(\mathrm{n}=8)$ and without DT $(n=5)$.

In the absolute spectral power analysis, high frequency power, particularly beta-3 power in the left frontal (Fp1 and F3), and parietal (Pz) areas were relatively lower in patients with DTs than in those without DTs $(P<0.05)$ The relative beta-3 powers of the left cortical areas ( $F 3, T 3, C 3$, and P3) were lower in the DTs group (Figure $3 \mathrm{~A}$ ). However, the beta-3 power of the right hemisphere and other ranges of spectral powers were not significantly different between the two groups $(P<0.05)$.

In the spectral power ratios, the central $(\mathrm{Cz})$ and occipital (01 and 02$)$ theta to alpha ratios (TAR) of DT patients were higher than those of non-DT patients $(P<0.05)$ (Figure $2 B)$.

\section{Discussion}

In this study, we compared the relative and absolute spectral power patterns across frequency bands between AWS patients in the acute period (within 48 hours after AWS) and age- and sex-matched healthy controls. In addition, we investigated whether spectral characteristics from early standard EEG are helpful for predicting and identifying patients at risk of developing DTs. The main findings of the current study were as follows: 1) Clinical and alcohol-related variables were comparable between AWS patients with or without DTs. 2) In QEEG analysis, AWS patients demonstrated higher relative delta, theta, and all range of beta power than did the healthy control group, whereas alpha power was lower in the AWS group. The spectral differences between the groups were predominant in the frontal area (except alpha-2 power, which was lower in almost all brain areas). 3) The absolute and relative spectral analysis showed that the high-frequency beta band power, specifically beta-3 $(20-30 \mathrm{~Hz})$, was lower in patients with than in patients without DTs. These differences were recorded with a certain topographic dominance in the left frontal cortical areas. Additionally, in terms of the spectral power ratios, patients with DTs had higher TAR than those without DTs in the central area.

Our observation of increased beta power and decreased alpha power in alcohol-withdrawal patients, as compared to healthy controls, was consistent with previous findings of EEG abnormalities in the alcohol- 
withdrawal state. Most previous studies investigating alcohol-dependence showed EEG abnormalities, such as generalized reduction of alpha rhythm and increased power in the delta, theta, and beta activities ${ }^{14}$. Compared with these previous results, our results expand the understanding of association between alcohol use and EEG characteristics, considering that alcohol-withdrawal syndromes are a part of alcohol-dependence. Rebound hyperexcitability, driven by abrupt cessation from chronic alcohol exposure, might affect neuroelectric activity in the central nervous system. Changes in the glutamate/GABA balance occurs during alcohol-withdrawal periods ${ }^{15}$. In addition, increased beta power might reflect increasing cortical hyperexcitability after seizure as well as an alcohol-withdrawal state, resulting from an imbalance between excitatory and inhibitory neurons. This speculation is supported by the mechanisms for the generation of beta oscillations, which involves the balance between networks of excitatory pyramidal cells (AMPAergic) and inhibitory interneurons (GABAergic) ${ }^{16}$. The cumulative neurophysiological effects of alcohol consumption on the brain might be suggestive of our findings. Increased absolute power in the beta frequency range of $12.5-20 \mathrm{~Hz}$ has been observed in alcoholdependence, over all brain locations, but prominently in the central brain region, in a previous study ${ }^{17}$. Another study showed alcohol-dependence was positively correlated with absolute high beta and gamma power $(20-40 \mathrm{~Hz})$ in the left fronto-central-parietal leads on EEG ${ }^{18}$.

Alpha power has been reported to be linked with cortical arousal level and cognitive and memory performance ${ }^{19,20}$. In particular, the slow alpha frequency $(8-10 \mathrm{~Hz}$; alpha-1) is related to attentional demands, whereas the fast alpha frequency $(10-12 \mathrm{~Hz}$; alpha-2) mediates semantic memory and stimulus-related aspects ${ }^{21}$. The frontal cortex (particularly the left inferior frontal gyrus), plays a role in supporting cognitive functions that are not only specific to language, as it has many afferent and efferent connections to all other neocortical regions (i.e., the parietal, temporal, and occipital regions, as well as to the cingulate, limbic, and basal ganglia structures ${ }^{22}$. The prefrontal cortex is thus the only cortical area interacting with all four sensory modalities received from olfactory sensation ${ }^{20}$. Alcoholism may particularly affect frontal cognitive function. In various brain imaging studies, chronic alcohol intake results in reduction of regional cerebral blood flow impairment, affecting the function of cerebral tissue in the medial frontal region, decreasing tissue metabolic rates, and affecting neurophysiology ${ }^{21,23}$. Alcoholdependence was associated with reduced absolute power and lower voltage of the alpha frequency in our study, which might reflect alcohol-related attentional, stimulus-reactive, and cognitive dysfunctions, as compared to in healthy controls, which was consistent with previous results about alcohol-dependence and electric deflection in the post-seizure state ${ }^{24,25}$. Taken together, the differential dominance in the frontal area between the AWS patients and the healthy control group can be explained by a strong connection of alcoholism to frontal lobe pathology and the post-seizure state ${ }^{23}$.

Studies on QEEG analysis in DTs, particularly in the alcohol-withdrawal state, remain scarce. Our study highlighted that specific EEG characteristics could be a significant predictor of DTs, based on a direct comparison of EEG data obtained in the acute period post-AWS. We found that the AWS group without DTs had higher absolute and relative power in the beta-3 range than did the AWS group with DTs. Although we did not perform cognitive assessment, cognitive deterioration associated with DTs could 
also be an explanation for the lower beta power in the AWS patients with DTs, given that lower beta power has negative effects on memory processing, such as episodic memory encoding and retrieval ${ }^{26}$. Patients with alcohol-dependence with DTs had worse intellectual functioning, which was clearly observed in terms of attention and productive visual-motor coordination as compared to such patients without DTs 27.

Findings regarding beta activity related to cognitive activity have been less consistent. However, the beta3 frequency, ranging from 20 to $30 \mathrm{~Hz}$, has specifically been associated with cognitive processes, such as semantic speech retrieval ${ }^{28}$, prosodic aspects ${ }^{29}$, and working memory ${ }^{30}$. It has been proposed that highfrequency band power, not only beta-3, but also gamma activity, is an indicator of cognitive activity and that coherent oscillations in this frequency range allow the binding of distant brain regions that are necessary to allow cognitive experience ${ }^{31}$. Visual or auditory stimulation and cognitive activities suppress brainwaves while increasing the power of the high-frequency beta and gamma bands ${ }^{32}$. Hence, the decreased high beta (specifically beta-3) power in the DT group may reflect cognitive dysfunction that precedes the development of DTs. This suggests that beta-3 power could be used as a predictive factor for the development of DTs during alcohol withdrawal. Interestingly, the difference in beta power between patients with and without DTs was prominent in the left hemisphere. While the reasons for asymmetry of EEG spectral power remain unclear, our finding may be in line with studies indicating that the mirrored reduction of left and right asymmetry might depend on the specific cognitive domain, such as verbal (left) and visuospatial (right) functions ${ }^{25,26}$. The frontal lobe, particularly the left frontal gyrus, is involved in functions such as creative thinking, planning of future actions, decision-making, artistic expression, aspects of emotional behaviour, and spatial working memory ${ }^{22}$. It has been reported that an increased high beta frequency band $(22-30 \mathrm{~Hz}$ ) induced by an epileptic drug (levetiracetam) was correlated with better neuropsychological measures in patients with epilepsy ${ }^{33}$. Enhancing activities of the neuronal networks in the prefrontal cortex and left hippocampus also correlates with an increased beta-3 band.

Another index used to explore the difference in EEG characteristics for predicting alcohol-withdrawal delirium is the frequency band ratio. Our results showed an increased TAR in the central area in patients who developed DTs. The reverse metric of the TAR, the alpha/theta ratio (ATR), has been used as a marker of functional connectivity and performance enhancement, and is used to discriminate individuals with probable Alzheimer's disease from healthy older controls. Increased frontal-midline theta is related to learning in a task and during performance of an attention-demanding procedure. The theta frequency has been associated with long-range functional interactions in working memory. The TAR is known to change with age and cognitive ability, even in healthy individuals. One study reported that the relationship between cognitive ability and the TAR was age-dependent, and that cognitive performance at the $C Z$ midline location predicted the TAR ${ }^{34}$. Another study showed that the TAR was increased relative to controls in older adults with amnesic mild cognitive impairment ${ }^{35}$. Alpha/theta neurofeedback training has also been shown to have clinical benefits in the treatment of alcoholism and addiction ${ }^{36,37}$. An increased TAR in patients with AWS, specifically in those who progressed to DTs, might indicate addiction 
and preceding cognitive impairment, which was previously found to be associated with Alzheimer's disease ${ }^{29}$.

The current study had several limitations. First, the study sample was small, and there might have been a selection bias, since this study was performed at a single center. Unexpectedly, most of the subjects who were initially enrolled were subsequently excluded because of structural brain lesions on neuroimaging, to avoid interference on EEG. Most of our subjects had brain lesions in cerebral cortex because alcohol intoxication is one of the strongest predictors of traumatic brain injury and a greater vascular burden ${ }^{38}$. Second, all subjects were male, implying that it may be difficult to generalize this finding to females. Third, we did not perform a neuropsychiatric test to identify delirium. Further studies using neuropsychiatric scores and QEEG findings (for example, cognitive deviation to the left and right cortical function or changes in degree between groups). However, this study had several strengths. This study was performed according to a strict protocol, under well-controlled circumstances and time-lines. Furthermore, no previous report has used QEEG to predict alcohol-related DTs, particularly after AWS, which is the severe state of alcohol-withdrawal.

\section{Conclusion}

Considering the broader economic and health care burden and mortality related to DTs, early identification of patients at risk for developing delirium tremens is essential for early intervention, to stop the cascades of negative outcomes. QEEG might be a helpful tool for detecting early changes for development of DTs in alcohol-withdrawal patients and could help physicians to identify patients with AWS who are at high risk of developing DTs during an alcohol-dependence period.

\section{Methods}

\section{Participants}

We retrospectively reviewed the electronic medical records of all patients who were diagnosed with alcohol-withdrawal seizure at admission to two university hospitals (Hallym University Hangang and Dongtan Sacred Heart Hospital) between March 2018 and December 2020. We enrolled 38 patients aged 19 years or older who underwent initial neuroimaging, such as computed tomography and/or magnetic resonance imaging, and EEG, within 48 hours after seizure, in the intensive care unit. The diagnosis of AWS and DTs was based on the Diagnostic and Statistical Manual of Mental Disorders (DSM-5) ${ }^{39}$.

We excluded patients with structural brain lesions on brain imaging that could confound EEG findings 40 and those in whom it was not possible to evaluate DTs due to underlying language disturbance, hearing difficulty, or cognitive impairment. After excluding 25 patients, 13 patients were finally analysed (Fig. 1).

For the comparison of EEG between the healthy population and patients, we selected data of age- and sex-matched healthy adults from the big data collected during the development of the EEG spectral 
analysis software iSyncBrain® (iMediSync, Inc., Korea).

The informed consents were obtained from all subjects. This study was approved by the Institutional Review Board (IRB) of Hallym University Medical Center (IRB No. HG2019-027 and DT2021-018). All methods were performed in accordance with the relevant guidelines and regulations

\section{Clinical assessment}

Clinical variables included age, sex, amount of alcohol consumption, and duration of alcohol intake for all patients. After admission to the ICU, blood samples were collected for assessment of white blood cell count, mean corpuscular volume, platelet count, prothrombin time and haemoglobin, albumin, vitamin B12, folic acid, aspartic acid transaminase, alanine aminotransferase, total bilirubin, direct bilirubin, $\mathrm{Y}^{-}$

glutamyl transferase, blood nitrogen urea, total cholesterol, ammonia, prolactin, and neuron-specific enolase levels. We also assessed the duration (days) of recovery of consciousness from seizure onset.

\section{Electroencephalogram recording}

All patients underwent EEG recording within $48 \mathrm{~h}$ after clinical seizure. Electrodes were placed according to the International 10-20 System. Patients were relaxed during the recording. Nineteen channels, with a common average reference montage, were used for recording, i.e., Fp1, F3, C3, P3, Fp2, F4, C4, P4, F7, T3, $\mathrm{T} 5, \mathrm{O} 1, \mathrm{~F} 8, \mathrm{~T} 4, \mathrm{~T} 6, \mathrm{O} 2, \mathrm{Fz}, \mathrm{Cz}$, and Pz. EEG recordings were conducted for at least 20 minutes, of which at least 5 minutes of awake EEG were included for each patient. The circumstances for recording and sources of artifacts were identical for all patients and were controlled during the examination in the intensive care unit. Two neurologists $(\mathrm{H}$. Mo and $\mathrm{H}-\mathrm{J} \mathrm{Im})$ selected 5 min of continuous eye-closed and artifact-free awake EEG data for further analysis.

\section{Data processing and analysis}

The selected EEG data were high-pass filtered above $1 \mathrm{~Hz}$, low-pass filtered below $45 \mathrm{~Hz}$, and recomputed to obtain a common average reference, offline. In addition to visual inspection, using advanced mixtureindependent component analysis (amICA), transient and stationary artifact components originating from eye movement, muscle, or heartbeat were removed. After cleansing the EEG signals, absolute and relative sensor-level analysis using a spectopo function in EEGLAB was performed for the following eight spectral bands: $\delta$ (1-4 Hz), $\theta(4-8 \mathrm{~Hz})$, a1 (8-10 Hz), a2 (10-12 Hz), $\beta 1$ (12-15 Hz), $\beta 2$ (15-20 Hz), $\beta 3(20-30 \mathrm{~Hz})$, and $\mathrm{Y}(30-45 \mathrm{~Hz})^{41,42}$.

Source reconstructions were performed using the standardized low-resolution brain electromagnetic topography (sLORETA) plugin, using a Colin 27 Head model with 68 region-of-interest segmentations based on the Desikan and Killiany atlas ${ }^{43,44}$.

All the preprocessing steps, de-noising using amICA, sensor-level feature extraction, source-level feature extraction, and generation of topomap images were performed on iSyncBrain ${ }^{\circledR}$ (iMediSync, Inc., Seoul, Korea) (https://isyncbrain.com/). Colour scale bar means difference of the interested group to the other 
group. In Figure 2, topomap showing the differences between the patients and control group, going toward the red side indicates that the group of interest (the patient group) has a relatively lower band frequency than the other group (the control group). Conversely, based on the colour bar, going toward the blue side represents the higher band frequencies. In Figure 3, topomap showing the differences between DT (-) and DT (+) group, going toward the red side indicates that the interested group (DT(+)) has a relatively higher band frequency than the other group (DT(-)). Conversely, based on the colour bar, going toward the blue side represents the lower band frequencies. $P$ values toward the red side indicate statistically significant $(P<0.05)$.

The analysis protocol has been documented in previous reports ${ }^{11,45}$.

\section{Statistical analysis}

Continuous variables with normal distributions are presented as mean \pm standard deviation, while those with non-normal distributions are presented as medians (interquartile ranges).

We compared baseline demographic information and risk factor profiles of patients with DT. We used the Mann-Whitney test for comparison of continuous variables and Fisher's exact test for comparison of categorical variables. All statistical analyses in the current study were performed using SPSS (version 24.0; IBM SPSS, Chicago, IL, USA). Statistical significance was considered at $P<0.05$.

Statistical analyses of the EEG features were performed using MATLAB (R2017b, MathWorks, Inc.). Statistical significance was assumed at $P<0.05$, and statistical analysis was performed automatically using the iSyncBrain ${ }^{\circledR}$ program.

\section{Declarations}

\section{Acknowledgements}

This research was supported by the Hallym University Research Fund 2019 (HURF-2019-60).

\section{Author Contributions}

$\mathrm{H}-\mathrm{J} \mathrm{Mo}, \mathrm{JE}$ Yoon and H-J Im contributed to the analysis and interpretation of data, and drafted the manuscript; DW Kim contributed to the data acquisition and interpretation, and revised the manuscript; JE Yoon interpreted results and revised the manuscript. H-J Im conceptualized and designed the study, analyzed and interpreted the data, and revised the manuscript.

\section{Competing interests}

The authors declare no competing interests.

\section{References}


1. Hillbom M, Pieninkeroinen I, Leone MJCd. Seizures in alcohol-dependent patients. 2003; 17 (14): 1013-1030.

2. Nutt D. Alcohol and the brain: pharmacological insights for psychiatrists. The British Journal of Psychiatry. 1999; 175 (2): 114-119.

3. Mennecier $\mathrm{D}$, Thomas $\mathrm{M}$, Arvers $\mathrm{P}$, et al. Factors predictive of complicated or severe alcohol withdrawal in alcohol dependent inpatients. Gastroentérologie clinique et biologique. 2008; 32 (8-9): 792-797.

4. Mainerova B, Prasko J, Latalova K, et al. Alcohol withdrawal delirium-diagnosis, course and treatment. Biomedical Papers of the Medical Faculty of Palacky University in Olomouc. 2015; 159 (1).

5. Wright T, Myrick H, Henderson S, Peters $\mathrm{H}$, Malcolm R. Risk factors for delirium tremens: a retrospective chart review. The American journal on addictions. 2006; 15 (3): 213-219.

6. Coleman MP, Quaresma M, Berrino F, et al. Cancer survival in five continents: a worldwide populationbased study (CONCORD). The lancet oncology. 2008; 9 (8): 730-756.

7. Eyer $\mathrm{F}$, Schuster T, Felgenhauer $\mathrm{N}$, et al. Risk assessment of moderate to severe alcohol withdrawalpredictors for seizures and delirium tremens in the course of withdrawal. 2011; 46 (4): 427-433.

8. Lee JH, Jang MK, Lee JY, et al. Clinical predictors for delirium tremens in alcohol dependence. Journal of gastroenterology and hepatology. 2005; 20 (12): 1833-1837.

9. Foy A, Kay J, Taylor AJQmjotAoP. The course of alcohol withdrawal in a general hospital. 1997; 90 (4): 253-261.

10. Ferguson JA, Suelzer CJ, Eckert GJ, Zhou X-H, Diffus RSJJogim. Risk factors for delirium tremens development. 1996; 11 (7): 410-414.

11. Baik K, Kim SM, Jung JH, et al. Donepezil for mild cognitive impairment in Parkinson's disease. Scientific reports. 2021; 11 (1): 1-9.

12. Koponen H, Partanen J, Pääkkönen A, Mattila E, Riekkinen PJJoN, Neurosurgery, Psychiatry. EEG spectral analysis in delirium. 1989; 52 (8): 980-985.

13. Van Der Kooi AW, Zaal IJ, Klijn FA, et al. Delirium detection using EEG. 2015; 147 (1): 94-101.

14. Funderburk W. Electroencephalographic studies in chronic alcoholism. In: proceedings from the Electroencephalography and Clinical Neurophysiology; 1949.

15. Brousse G, Arnaud B, Vorspan F, et al. Alteration of glutamate/GABA balance during acute alcohol withdrawal in emergency department: a prospective analysis. Alcohol and alcoholism. 2012; 47 (5): 501-508.

16. Whittington MA, Traub RD, Kopell N, Ermentrout B, Buhl EH. Inhibition-based rhythms: experimental and mathematical observations on network dynamics. International journal of psychophysiology. 2000; 38 (3): 315-336.

17. Rangaswamy M, Porjesz B, Chorlian DB, et al. Beta power in the EEG of alcoholics. Biological psychiatry. 2002; 52 (8): 831-842. 
18. Ehlers CL, Phillips E, Gizer IR, Gilder DA, Wilhelmsen KC. EEG spectral phenotypes: Heritability and association with marijuana and alcohol dependence in an American Indian community study. Drug and alcohol dependence. 2010; 106 (2-3): 101-110.

19. Başar E. A review of alpha activity in integrative brain function: fundamental physiology, sensory coding, cognition and pathology. International Journal of Psychophysiology. 2012; 86 (1): 1-24.

20. Nauta WJ. The problem of the frontal lobe: A reinterpretation. Principles, Practices, and Positions in Neuropsychiatric Research. 1972: 167-187.

21. Adams KM, Gilman S, Koeppe RA, et al. Neuropsychological deficits are correlated with frontal hypometabolism in positron emission tomography studies of older alcoholic patients. Alcoholism: Clinical and Experimental Research. 1993; 17 (2): 205-210.

22. Tyler LK, Marslen-Wilson WD, Randall B, et al. Left inferior frontal cortex and syntax: function, structure and behaviour in patients with left hemisphere damage. Brain. 2011; 134 (2): 415-431.

23. Moselhy HF, Georgiou G, Kahn A. Frontal lobe changes in alcoholism: a review of the literature. Alcohol and alcoholism. 2001; 36 (5): 357-368.

24. Paulucio D, da Costa BM, Santos CG, et al. Acute ethanol and taurine intake affect absolute alpha power in frontal cortex before and after exercise. Neuroscience letters. 2017; 657: 5-10.

25. Ehlers CL, Phillips E. Association of EEG alpha variants and alpha power with alcohol dependence in Mexican American young adults. Alcohol. 2007; 41 (1): 13-20.

26. Nyhus E. Brain networks related to beta oscillatory activity during episodic memory retrieval. Journal of cognitive neuroscience. 2018; 30 (2): 174-187.

27. Dickov A, Vuckovic N, Martinovic-Mitrovic S, et al. Disorder verbal memory in alcoholics after delirium tremens. Eur Rev Med Pharmacol Sci. 2012; 16 (8): 1052-1060.

28. Shahin AJ, Picton TW, Miller LM. Brain oscillations during semantic evaluation of speech. Brain and cognition. 2009; 70 (3): 259-266.

29. Weiss S, Mueller HM. "Too many betas do not spoil the broth": the role of beta brain oscillations in language processing. Frontiers in psychology. 2012; 3: 201.

30. Pavlov YG, Kotchoubey B. EEG correlates of working memory performance in females. BMC neuroscience. 2017; 18 (1): 1-14.

31. Gross DW, Gotman J. Correlation of high-frequency oscillations with the sleep-wake cycle and cognitive activity in humans. Neuroscience. 1999; 94 (4): 1005-1018.

32. Marrufo MV, Vaquero E, Cardoso MaJ, Gomez CM. Temporal evolution of a and $\beta$ bands during visual spatial attention. Cognitive Brain Research. 2001; 12 (2): 315-320.

33. Park S-P, Kwon O-Y. Increased EEG current-source density in the high Beta frequency band induced by levetiracetam adjunctive therapy in refractory partial epilepsy. Journal of Clinical Neurology. 2009; 5 (4): 178-185.

34. Trammell JP, MacRae PG, Davis G, Bergstedt D, Anderson AE. The Relationship of Cognitive Performance and the Theta-Alpha Power Ratio Is Age-Dependent: An EEG Study of Short Term 
Memory and Reasoning during Task and Resting-State in Healthy Young and Old Adults. Front Aging Neurosci. 2017; 9: 364.

35. Bian Z, Li Q, Wang L, Lu C, Yin S, Li X. Relative power and coherence of EEG series are related to amnestic mild cognitive impairment in diabetes. Frontiers in aging neuroscience. 2014; 6: 11.

36. Peniston EG, Kulkosky PJ. Alcoholic personality and alpha-theta brainwave training. Medical Psychotherapy: An International Journal. 1990.

37. Gruzelier J. A theory of alpha/theta neurofeedback, creative performance enhancement, long distance functional connectivity and psychological integration. Cognitive processing. 2009; 10 (1): 101-109.

38. Weil ZM, Corrigan JD, Karelina K. Alcohol use disorder and traumatic brain injury. Alcohol research: current reviews. 2018; 39 (2): 171.

39. Asken MJ, Grossman D, Christensen LW. American Psychiatric Association. Diagnostic and Statistical Manual of Mental Disorders. Arlington, VA: American Psychiatric Pub-lishing, 2013. Archibald, Herbert C., and Read D. Tuddenham."Persistent Stress Reac-tion after Combat: A 20-Year Follow-Up." Archives of General Psy. Therapy. 2007; 45 (10): 2317-2325.

40. Duncan JS. Imaging and epilepsy. Brain: a journal of neurology. 1997; 120 (2): 339-377.

41. Delorme A, Makeig S. EEGLAB: an open source toolbox for analysis of single-trial EEG dynamics including independent component analysis. Journal of neuroscience methods. 2004; 134 (1): 9-21.

42. Delorme A, Palmer J, Onton J, Oostenveld R, Makeig S. Independent EEG sources are dipolar. PloS one. 2012; 7 (2): e30135.

43. Holmes CJ, Hoge R, Collins L, Woods R, Toga AW, Evans AC. Enhancement of MR images using registration for signal averaging. Journal of computer assisted tomography. 1998; 22 (2): 324-333.

44. Desikan RS, Ségonne F, Fischl B, et al. An automated labeling system for subdividing the human cerebral cortex on MRI scans into gyral based regions of interest. Neuroimage. 2006; 31 (3): 968-980.

45. Kang SW, Choi SH, Jeong JH, et al. Difference of quantitative EEG between Alzheimer's disease (AD) dementia and non-dementia AD: Neuroimaging/New imaging methods. Alzheimer's \& Dementia. 2020; 16: e044300.

\section{Figures}



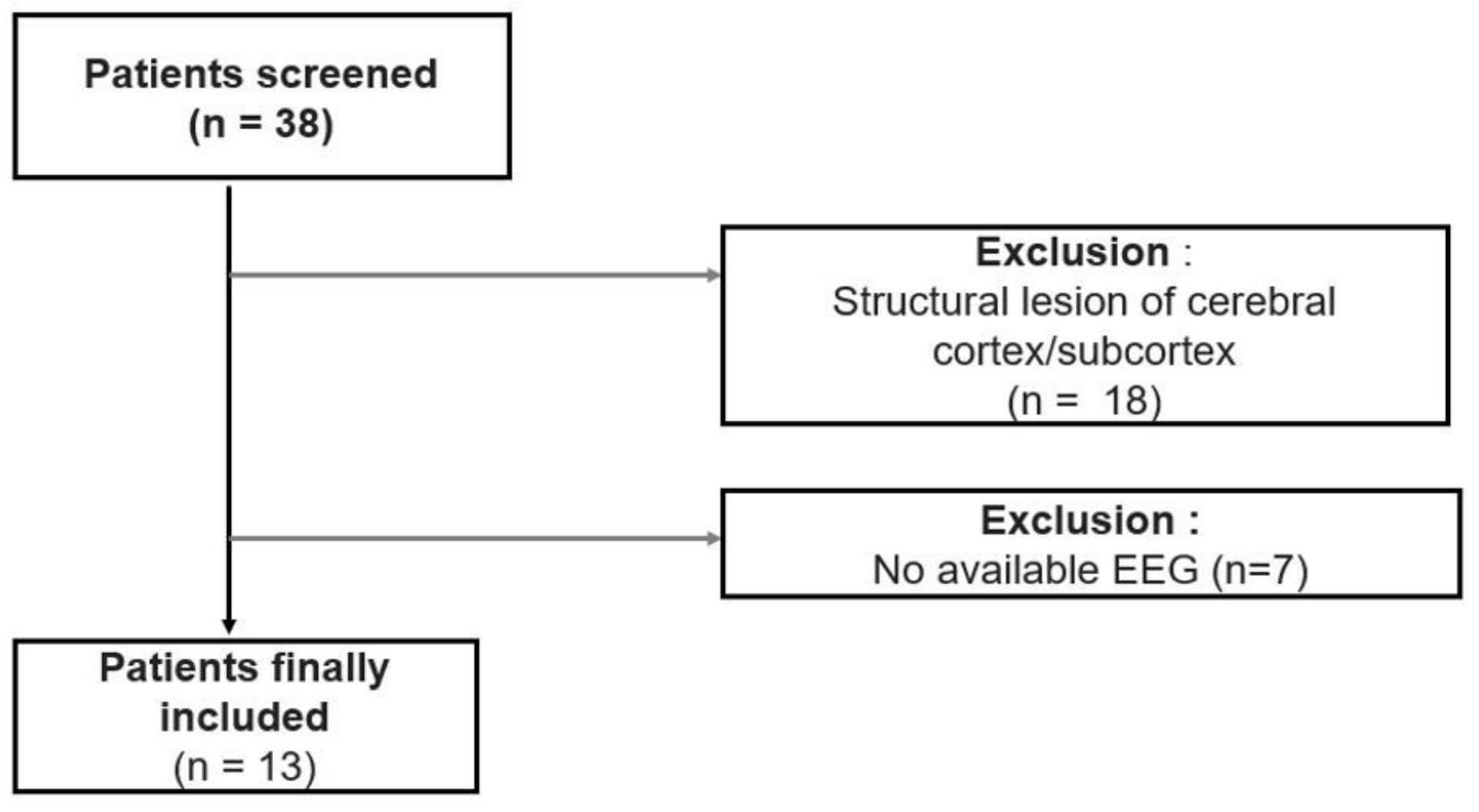

Figure 1

Flow chart of patient selection

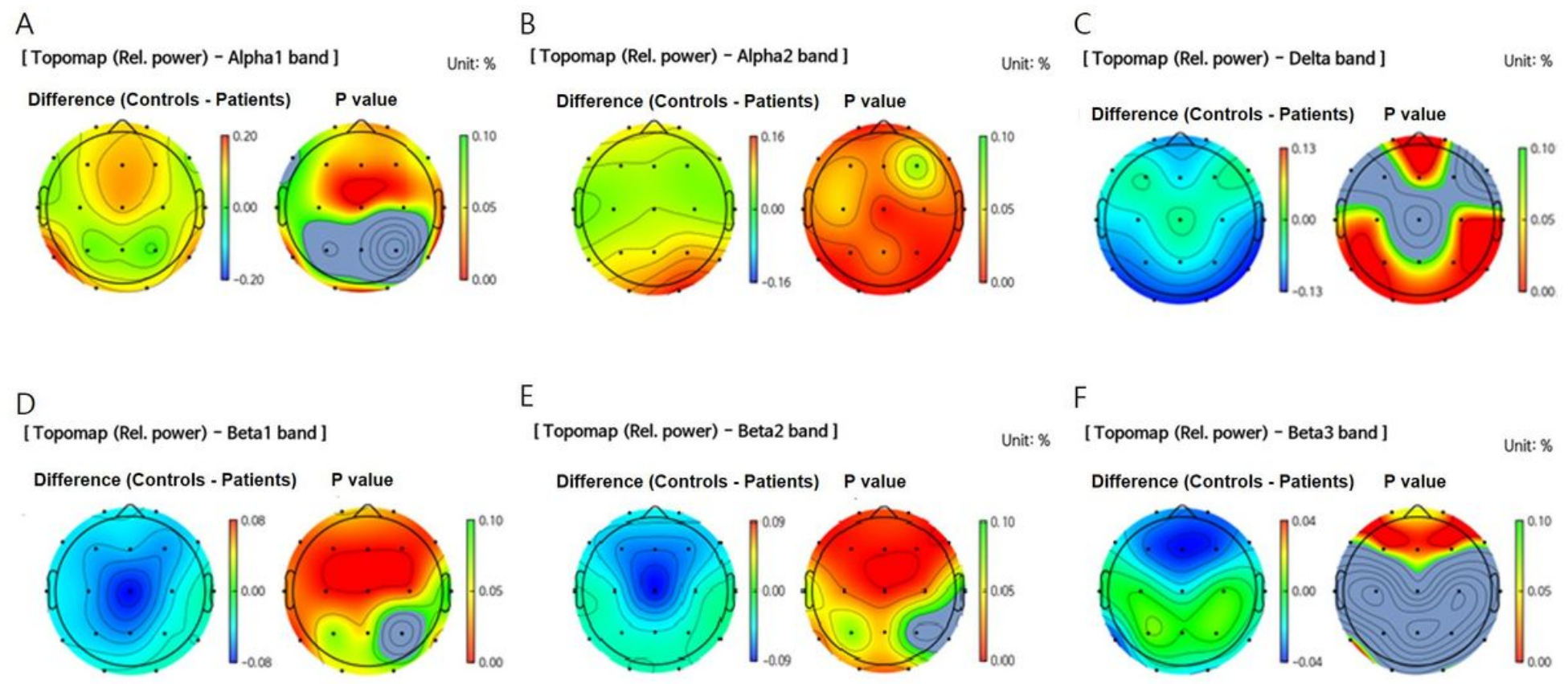

Figure 2

Topomap of comparison of relative spectral power bands between the patients after AWS and healthy controls 
Topomap showing the differences between the patients and control group, going toward the red side indicates that the group of interest (the patient group) has a relatively lower band frequency than the other group (the control group). Conversely, based on the colour bar, going toward the blue side represents the higher band frequencies. $P$ values toward the red side indicate statistically significant $(P<0.05)$.
(A) Alpha-1 band $(8-10 \mathrm{~Hz})$,
(B) Alpha-2 band (10-12 Hz),
(C) Delta band (1-4 Hz),
(D) Beta-1 band (12-15
$\mathrm{Hz}),(\mathrm{E})$ Beta-2 band (15-20 Hz), (F) Beta3 band (20-30 Hz). AWS; Alcohol withdrawal seizure

A

Topomap (Rel.power) -Beta3
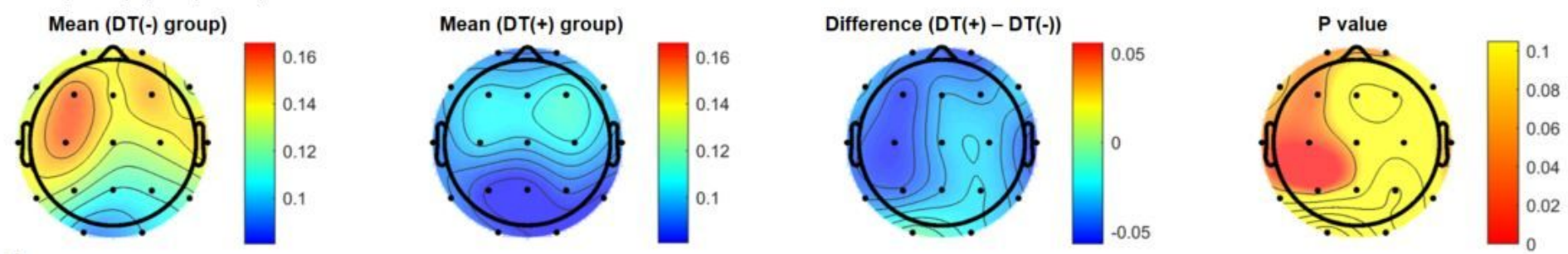

B

Theta/Alpha ratio (TAR)

Mean (DT(-) group)
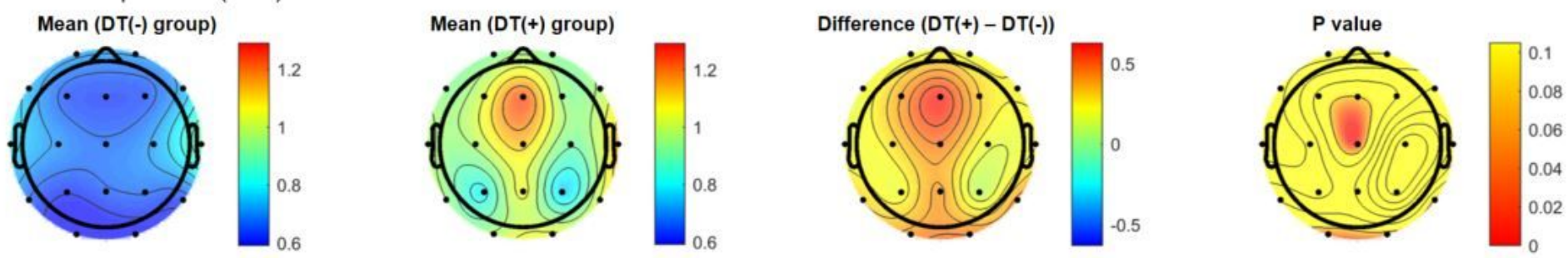

Figure 3

\section{Topomap of comparison of relative spectral power band and theta/alpha band ratio between the patients with and without delirium tremens}

Topomap showing the differences between DT (-) and DT (+) group, going toward the red side indicates that the interested group (DT(+)) has a relatively higher band frequency than the other group (DT(-)). Conversely, based on the colour bar, going toward the blue side represents the lower band frequencies. Topomap of $\mathrm{p}$-value from comparison between groups toward the red side indicate statistically significant $(p<0.05)$.

(A) Beta3 band (20-30 Hz), (B) Theta/alpha band power ratio. DT; Delirium tremens, +; with, -; without 